\title{
Indolent T-cell lymphoproliferative disorder of the stomach successfully treated by radiotherapy
}

\author{
Naoki Takahashi, ${ }^{1)}$ Kunihiro Tsukasaki, ${ }^{1)}$ Mika Kohri, ${ }^{1)}$ Yu Akuzawa, ${ }^{1)}$ Tsuyoshi Saeki, \\ Daisuke Okamura, ${ }^{1)}$ Maho Ishikawa, ${ }^{1)}$ Tomoya Maeda, ${ }^{1)}$ Nobutaka Kawai, ${ }^{1)}$ Akira Matsuda, \\ Eiichi Arai, ${ }^{2)}$ Shin Arai, ${ }^{3)}$ Norio Asou ${ }^{1)}$
}

Successful treatment of indolent T-cell lymphoproliferative disorder of the gastrointestinal tract (ITLPDGI) by chemotherapy is rare and watchful waiting is often performed for asymptomatic patients. We report a case of ITLPDGI successfully treated by involved field radiotherapy (IFRT). The patient presented with slow ITLPDGI localised to the stomach with mild symptoms. IFRT (30 Gy/20f) was administered, after which endoscopy revealed resolution of lesions and blood vessel appearance, and absence of proliferating abnormal lymphocytes was confirmed by biopsy. The patient remains lymphoma-free 1 year posttreatment. Although long-term follow-up and additional cases are essential for the evaluation of IFRT as a treatment option for localised ITLPDGL, complete remission after relatively low-dose IFRT is promising, particularly as this has been rarely achieved by chemotherapy.

Keywords: ITLPDGL, treatment, involved field radiotherapy, gastric cancer, lymphoproliferative disorder

\section{INTRODUCTION}

Peripheral T-cell lymphoma not otherwise specified (PTCL-NOS) is a non-Hodgkin lymphoma according to the World Health Organization (WHO) classification. ${ }^{1}$ It is the most frequent mature T- and NK-cell neoplasm, and represents a heterogeneous group of nodal and extra-nodal mature T-cell lymphomas that do not belong to any other recognised mature T-cell lymphoma subtype. Most patients with PTCLNOS present with peripheral lymph node involvement, but any extra-nodal site may be affected. ${ }^{1}$ PTCL-NOS is a highly aggressive lymphoid neoplasm with a poor response to treatment and exhibits frequent relapse even after remission. A high international prognosis index or prognostic index score for peripheral T-cell lymphoma is associated with a poor prognosis after treatment and a large number of transformed T-cells, which are more resistant to cytotoxic therapy than B-cells, possibly due to their high expression of multidrug resistant genes, is also related to a poor prognosis. ${ }^{2,3}$ Recently, PTCL-NOS of several extra-nodal sites, including the gastrointestinal (GI) tract, thyroid, liver and spleen with or without lymph nodes, have been reported by us and others as presenting an indolent clinical course without treatment or long-term survival after treatment with persistent local disease despite treatment. ${ }^{4,5}$ In the $4^{\text {th }}$ revised WHO classification of lymphoid malignancies, a provisional entity of "indolent T-cell lymphoproliferative disorder of the gastrointestinal tract" (ITLPDGI) was described, ${ }^{6,7}$ distinct from other aggressive T-cell lymphomas of the GI tract.

We report a patient with primary gastric ITLPDGI positive for CD3/CD5/CD8/TIA-1/Granzyme and negative for CD4/CD56/EBER who was successfully treated by low-dose radiotherapy and achieved stable complete remission.

\section{CASE REPORT}

A woman in her late 70s presented with mild epigastralgia and weight loss in October 2015. In October 2016, she came to our hospital for abnormal findings on upper gastrointestinal endoscopy (GIS). Physical examination revealed nothing in particular. Blood cell counts were within normal limits, and no increase in lactose dehydrogenase or soluble

\footnotetext{
Received: June 11, 2019. Revised: January 23, 2020. Accepted: February 18, 2020. Onlune Published: March 28, 2020

DOI: $10.3960 /$ jslrt. 19022

${ }^{1)}$ Department of Hematology, Comprehensive Cancer Center, International Medical Center, Saitama Medical University, Hidaka, Saitama, Japan, ${ }^{2}$ Department of Pathology,

Comprehensive Cancer Center, International Medical Center, Saitama Medical University, Hidaka, Saitama, Japan, ${ }^{3)}$ Department of Gastroenterology, Comprehensive Cancer Center, International Medical Center, Saitama Medical University, Hidaka, Saitama, Japan.

Corresponding author: Naoki Takahashi, Department of Hematology, Comprehensive Cancer Center, International Medical Center, Saitama Medical University, 1397-1, Yamane,

Hidaka, Saitama 350-1298, Japan. Email: ntakashi@saitama-med.ac.jp

Copyright (C) 2020 The Japanese Society for Lymphoreticular Tissue Research

(cc) BY-NC-SA This work is licensed under a Creative Commons Attribution-NonCommercial-ShareAlike 4.0 International License.
} 
IL-2 receptor was detected. Antibody for human T-lymphotropic virus type-1 was negative. GIS (Figure 1) revealed multiple erosions in the lower body and major and minor curvature of the stomach. On narrow band imaging, abnormal blood vessels with a so-called "tree-like appearance" that tapered to the erosion site were noted, suggesting superficial malignant lesions. Biopsy revealed dense proliferation of small-medium-sized lymphocytes with little atypia in the submucosal layer with some lymphoepithelial lesions (Figure 2). On immunohistochemical staining, the abnormal lymphocytes were CD3+, CD4-, CD5+, CD8+, CD43+, CD56-, TIA-1+, Granzyme+, TCR $\beta F 1+$ and TCRC $\gamma \mathrm{M} 1-$. The Ki-67 proliferation index was low $(\sim 10 \%)$. PCR demonstrated that the T-cell receptor $\gamma$ was rearranged and in situ hybridisation for EBER was negative in the gastric specimen. A diagnosis of primary gastric ITLPDG was made based on immunohistological findings and the slow clinical course. No abnormal findings were observed by colonoscopy. Moreover, no abnormal accumulation was detected by PET-CT in the stomach or other GI areas. We concluded that this patient had lymphoma in the stomach only (CS IA) with mild symptoms. For this reason, involved field radiotherapy (IFRT) (30 Gy/20f) was performed in March 2017. After radiotherapy, GIS revealed the disappearance of erosions and abnormal vessel appearance. Furthermore, gastric biopsy confirmed the absence of proliferation of smallmedium abnormal lymphocytes, demonstrating the complete remission of ITLPDGI. The patient is alive and has been lymphoma-free for more than 1 year according to computed tomography and GIS.

\section{DISCUSSION}

ITLPDGI is a disease newly added as a provisional entity in the 4th revised WHO classification. ${ }^{1,7}$ It is distinct from other aggressive T-cell lymphomas of the GI tract, including enteropathy-associated T-cell lymphoma, monomorphic epitheliotropic intestinal T-cell lymphoma, adult T-cell

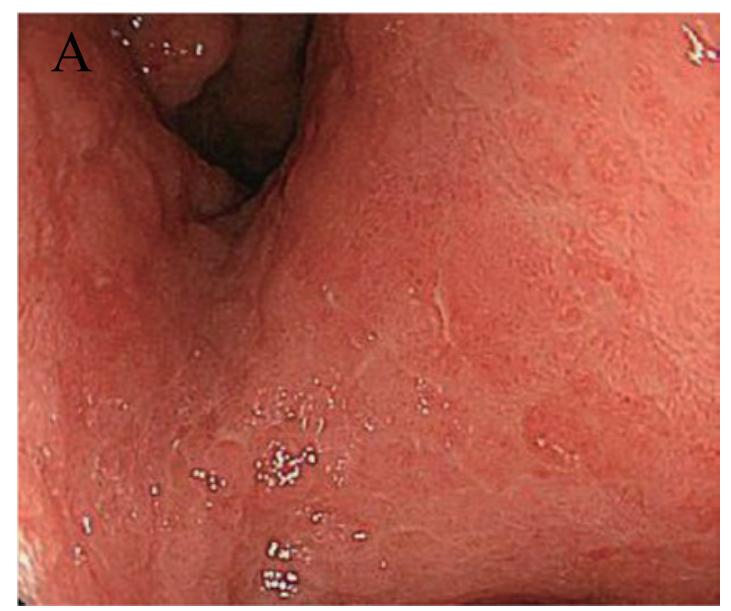

leukaemia/lymphoma, extranodal NK-/T-cell lymphoma, nasal type and PTCL-NOS. The review by Matani et al. ${ }^{8}$ of "Indolent T- and NK-lymphoproliferative disorders of the GI tract" included NK-cell lymphomatoid gastropathy and ITLPDGI. ${ }^{8,9}$ However, NK-cell lymphomatoid gastropathy was not included in the WHO classification of lymphoid malignancies because it was considered benign even though some cases persisted or relapsed..$^{9,10}$

ITLPDGI has been reported in many parts of the GI tract, frequently with multiple lesions in each case, and is characterised by the absence of lesions outside the GI tract. The most common site is the small intestine, followed by the large intestine. Macroscopic and endoscopic findings often include shallow erosions with erythema and multiple small polyps. Histopathological findings of typical ITLPDGI include dense infiltration of small-medium-sized lymphoma cells in the mucosal lamina propria without destruction of the mucosa. Enteropathy findings, such as villous atrophy, crypt hyperplasia and inflammatory cell infiltration, are sometimes observed, but a characteristic increase in intra-epithelial lymphocytes is usually not apparent. STAT3 abnormality was frequently observed in several PTCL entities. STAT3 SH2 domain mutation or activation was not observed upon first analysis, but recently, recurrent STAT3-JAK2 fusions have been found in ITLPDGI. ${ }^{11}$

Perry et al. recently reported a series of 10 ITLPDGI cases as PTCL-NOS localised in the GI tract with a non-progressive clinical course, irrespective of chemotherapy with or without autologous haematopoietic stem cell transplantation (AHSCT). Patients were a median of 48 years old and were predominantly male, with frequent symptoms including chronic abdominal pain and diarrhoea. ${ }^{7}$ Lesions involved the oral cavity, oesophagus, stomach, small intestine and colon. Infiltrates were dense but non-destructive and composed of small, mature-appearing lymphoid cells. Eight cases were CD4-/CD8+, one was CD4+/CD8-and the other was CD4-/CD8-. T-cell receptor $\gamma$ chain gene rearrangement analysis identified a clonal population in all 10 cases. Six

Fig. 1. Findings on upper GI endoscopy

$(A)$ Erosions in the lower body and major and minor curvature of the stomach

(B) Narrow band imaging revealed abnormal blood vessels (tree-like appearance) tapering to the erosion site 

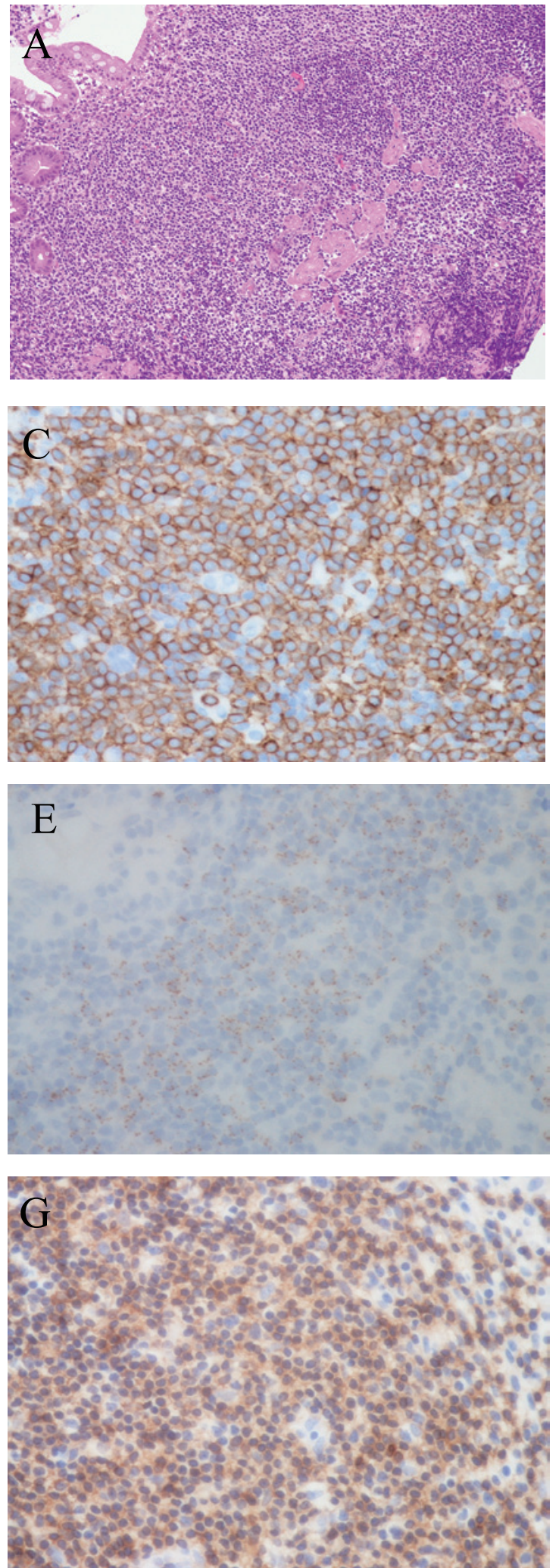
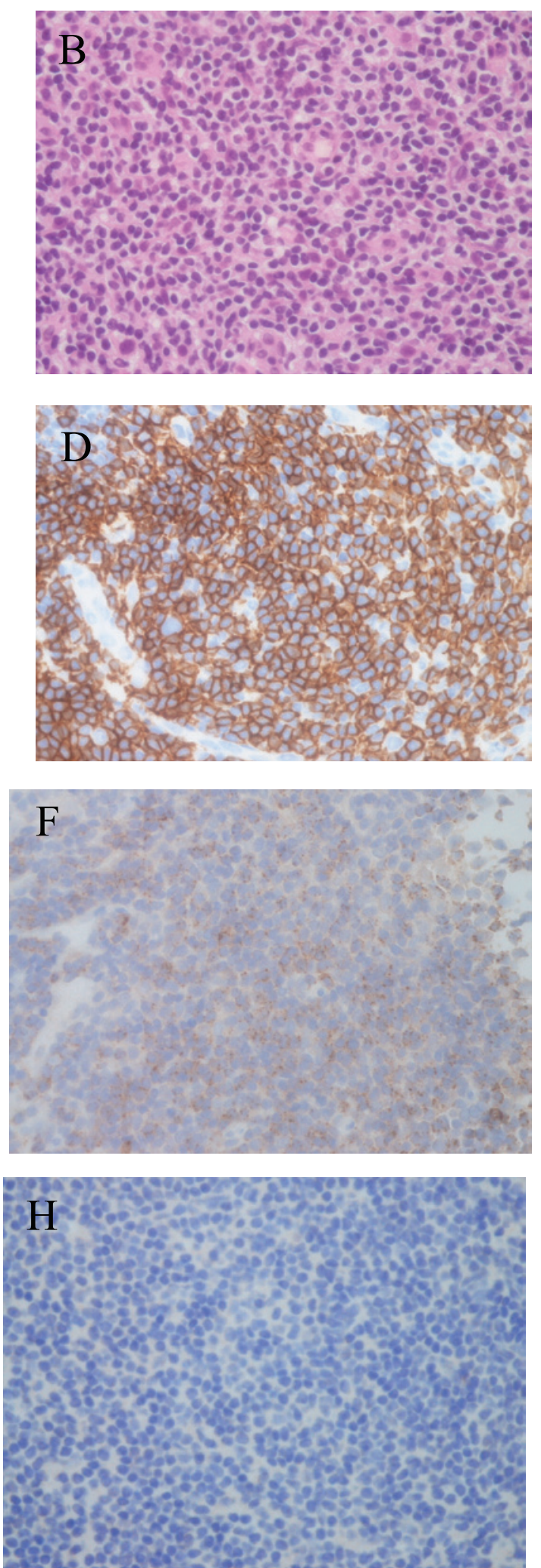

Fig. 2. Pathological findings

$(\boldsymbol{A})$ Dense proliferation of small to medium-sized lymphocytes is observed in the submucosal layer (haematoxylin and eosin, original magnification $\times 100)$; $(\boldsymbol{B})$ High magnification of the tumour (haematoxylin and eosin, original magnification $\times 400)$; $(\boldsymbol{C}$ $\boldsymbol{H})$ Immunohistochemical staining showing positivity for CD3 $(\boldsymbol{C})$, CD8 $(\boldsymbol{D})$, Granzyme B $(\boldsymbol{E})$, TIA-1 $(\boldsymbol{F})$ and TCR $\beta$ F1 $(\boldsymbol{G})$, and negativity for TCRC $\gamma \mathrm{M} 1(\boldsymbol{H})$ (immunoperoxidase, original magnification $\times 400)$. 
patients received chemotherapy with no response and four did not receive chemotherapy. After a median follow-up of 38 months (range, 9-175 months), nine patients were alive with persistent disease and one was free of disease after spontaneous regression.

Recognition of indolent PTCLs, such as ITLPDGI and primary thyroid PTCL, is important because the clinical course and treatment strategy are distinct from those for typical PTCL-NOS; however, the treatment for ITLPDGI has not yet been established. Although the clinical course is indolent by definition, the response to chemotherapy, including high-dose chemotherapy followed by AHSCT, is usually poor and lymphoma may persist with or without symptoms. Therefore, watchful waiting is a treatment option if patients are asymptomatic. In our case, because the lymphoma was localised only in the stomach with minor symptoms, IFRT of a relatively low dose (30 Gy) was selected, as in other cases of indolent T-cell lymphomas, such as primary PTCL-NOS of the thyroid, and significant single lesions of cutaneous T-cell lymphomas. This is the first case report of gastric ITLPDGI that was successfully treated by IFRT. Although long-term follow-up is essential for the evaluation of IFRT for localised ITLPDGL because of its long natural course, complete remission after relatively low-dose IFRT is promising as this is rarely achieved by chemotherapy. Further accumulation of cases of ITLPDGI is required to provide better treatment for patients with this rare entity.

\section{ACKNOWLEDGMENTS}

We thank Gillian Campbell, PhD, from Edanz Group for editing a draft of this manuscript.

\section{CONFLICTS OF INTEREST}

The authors declare that they have no conflicts of interest.

\section{REFERENCES}

1 Pileri SA, Ralfkiaer E, Weisenburger DD, et al. Peripheral T-cell lymphoma, not otherwise specified. In: World Health Organization Classification of Tumours of Haematopoietic and Lymphoid Tissues. $4^{\text {th }}$ ed, Lyon, IARC Press. 2009; pp. 306-309.
2 Vose J, Armitage J, Weisenburger D; International T-Cell Lymphoma Project. International peripheral T-cell and natural killer/T-cell lymphoma study: pathology findings and clinical outcomes. J Clin Oncol. 2008; 26 : 4124-4130.

3 Gallamini A, Stelitano C, Calvi R, et al.; Intergruppo Italiano Linfomi. Peripheral T-cell lymphoma unspecified (PTCL-U): a new prognostic model from a retrospective multicentric clinical study. Blood. 2004; 103 : 2474-2479.

4 Yoshida N, Nishikori M, Izumi T, et al. Primary peripheral T-cell lymphoma, not otherwise specified of the thyroid with autoimmune thyroiditis. Br J Haematol. 2013; 161 : 214-223.

5 Hayashi E, Takata K, Sato Y, et al. Distinct morphologic, phenotypic, and clinical-course characteristics of indolent peripheral T-cell lymphoma. Hum Pathol. 2013; 44 : 1927-1936.

6 Swerdlow SH, Campo E, Pileri SA, et al. The 2016 revision of the World Health Organization classification of lymphoid neoplasms. Blood. 2016; 127 : 2375-2390.

7 Perry AM, Warnke RA, Hu Q, et al. Indolent T-cell lymphoproliferative disease of the gastrointestinal tract. Blood. 2013; 122 : 3599-3606.

8 Matnani R, Ganapathi KA, Lewis SK, et al. Indolent T- and NK-cell lymphoproliferative disorders of the gastrointestinal tract: a review and update. Hematol Oncol. 2017; 35 : 3-16.

9 Takeuchi K, Yokoyama M, Ishizawa S, et al. Lymphomatoid gastropathy: a distinct clinicopathologic entity of self-limited pseudomalignant NK-cell proliferation. Blood. 2010; 116 : 5631-5637.

10 Mansoor A, Pittaluga S, Beck PL, et al. NK-cell enteropathy: a benign NK-cell lymphoproliferative disease mimicking intestinal lymphoma: clinicopathologic features and follow-up in a unique case series. Blood. 2011; 117 : 1447-1452.

11 Sharma A, Oishi N, Boddicker RL, et al. Recurrent STAT3$J A K 2$ fusions in indolent T-cell lymphoproliferative disorder of the gastrointestinal tract. Blood. 2018; 131 : 2262-2266. 